\title{
Performance of UDP and TCP Communication on Personal Computers
}

\author{
Andro Milanović, Siniša Srbljić, and Vlado Sruk
}

\begin{abstract}
The number of personal computers (PCs) in Internet based information systems has been rapidly increasing during the last few years. Designers are trying to improve the performance by choosing the appropriate hardware and software components of PCs that are running Internet based information systems. However, little research has been done to find how different hardware and software components of PCs affect the performance of the two most commonly used protocols for transferring data on Internet today, TCP and UDP.
\end{abstract}

This paper compares data transmission speed for three different operating systems: Windows 95, Windows NT 4.0 Workstation and Linux 2.1.132. Three different hardware configurations based on Intel $486 \mathrm{DX} 2$, Intel Pentium, and Intel Celeron $A$ processors are also compared. Finally, we show how data packet size affects the communication speed.

Index Terms - UDP, TCP, performance measure, personal computers.

\section{INTRODUCTION}

The Internet has shown amazing growth in recent years and its growth is still not slowing down. The number of personal computers and their role in the Internet have increased as well. Designers of PC-based Internet systems must make decisions based on a large number of various parameters. Some of the parameters designers have to consider are processor, memory, network card, LAN technology, operating system, and applications. It is hard to find research papers and performance results that present how these parameters affect the performance of Internet communication of personal computers.

The paper [2] gives performance results for Internet communication, but it focuses on RPC-based communication and Unix workstations. In this paper, we give the results for PCs and the two most commonly used protocols for transferring data on Internet today, TCP and UDP [3-4]. The first protocol is used for reliable communication, while the other protocol, UDP, shows better results in high-speed communication where a certain level of unreliability is acceptable.

We present the performance results for UDP and TCP communication speed as an extension to the paper [1]. The results for UDP and TCP in this paper were

A. Milanović, S. Srbljić and V. Sruk are with the School of Electrical Engineering and Computing, University of Zagreb, Zagreb, Croatia. e-mail: \{andro, sinisa, sruk\}@zemris.fer.hr obtained by measuring the data transmission speed between two PCs. We varied different hardware configurations of PCs, operating systems and message sizes to find how these three parameters affect data transmission speed.

In Section 2, we describe the measurement environment including the software package used in the measurement process. The performance measurement results for UDP are presented and discussed in Section 3. Section 4 describes the results of performance measurements for TCP. Final comments on the performance measurement results are given in Section 5.

\section{MEASUREMENT ENVIRONMENT}

We developed a small software package for the purpose of measuring the performance. The package consists of two programs: the Sender and the Receiver. There are two versions of the testing package; one version is intended for use on the Windows 95 and Windows NT 4.0 operating systems, while the other is used with Linux.

The Sender program sends data and the Receiver program receives data. The following three parameters of the Sender program are configurable: the amount of data, the sending speed, and the size of data load. The Receiver program counts the received data, because UDP does not guarantee delivery of the sent data.

All tests were performed on a local network using 10 $\mathrm{Mb} / \mathrm{s}$ Ethernet, and the test machines were always in the same segment. Since we chose the receiving speed as the performance measure, we used the fastest system available, a Celeron A 333 system, as the sending machine. A high-speed sending machine enabled us to find saturation speed of the receiving machines.

Three different hardware configurations of PCs were tested and each of them is briefly described in the Table 1. For the purpose of comparing different hardware parameters, all of these configurations were running the same operating system, Windows NT 4.0 Workstation.

Table 1: Hardware configurations of PCs

\begin{tabular}{|c|c:c:c|}
\hline \hline & Intel 486 DX2 & Intel Pentium & Intel Celeron A \\
\hline Processor speed $(\mathrm{MHz})$ & 66 & 133 & 300 \\
Cache size $(\mathrm{KB})$ & 128 & 256 & 128 \\
\hdashline RAM $(\mathrm{MB})$ & 20 & 32 & 128 \\
\hdashline Type of RAM & $70 \mathrm{~ns}$ & $60 \mathrm{~ns}, \mathrm{EDO}$ & PC-100 \\
\hdashline Bus & ISA & PCI & PCI \\
\hline \hline
\end{tabular}




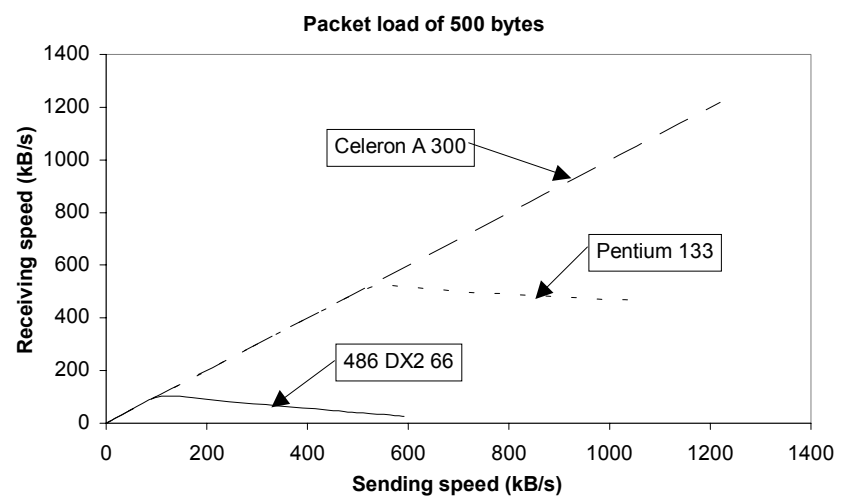

(a)

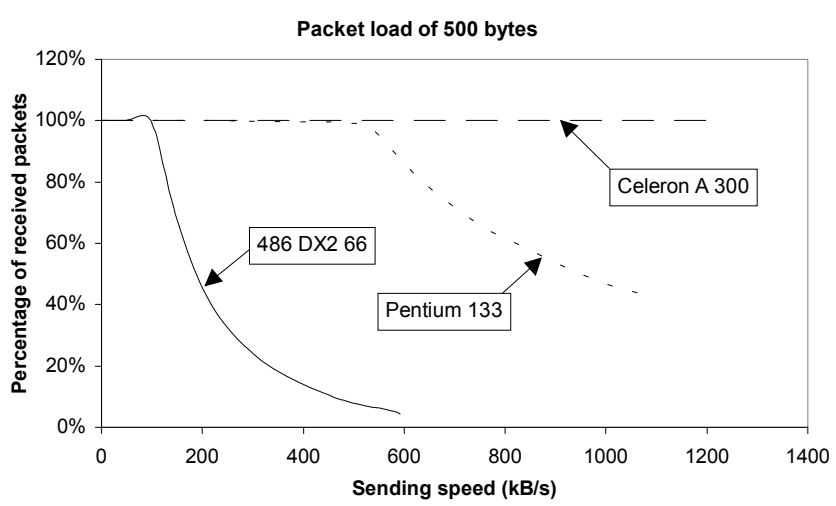

(b)

Figure 1: The comparison of UDP transmission speed for different hardware configurations and 500 bytes of packet load

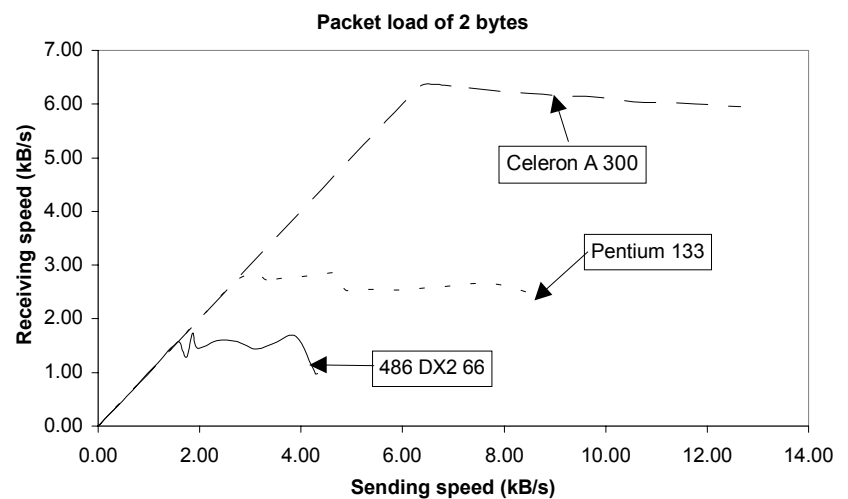

(a)

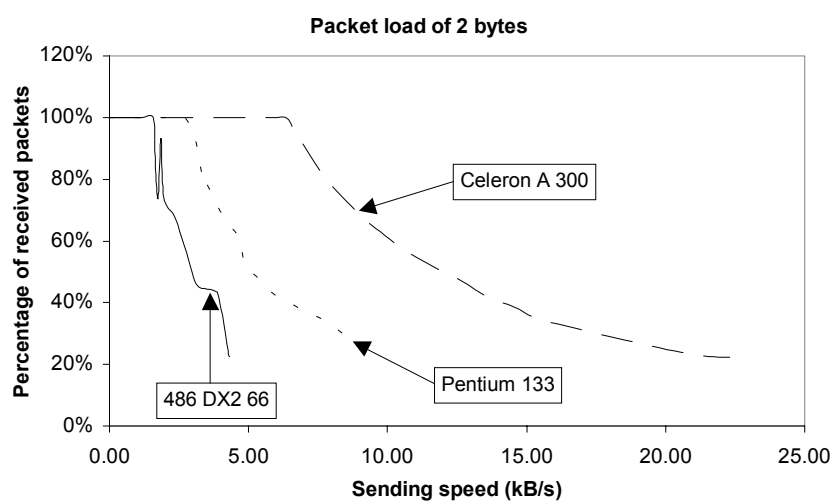

(b)

Figure 2: The comparison of UDP transmission speed for different hardware configurations and 2 bytes of packet load

We also performed tests with three different operating systems: Windows 95 OSR2, Windows NT 4.0 Workstation, and Linux 2.1.132. All operating systems were tested with the same hardware configuration, an Intel Pentium 133 system.

We used two data packet sizes in the measurements. One size was 500 bytes, representing an average data packet used on Internet in various protocols such as HTTP [5]. The other data packet size we used was two bytes per message and it presents how the system handles small data packets that carry either confirmation messages or some small data chunks.

\section{UDP MEASUREMENT RESULTS}

Figures 1-4 present the results of measurements of data transmission speed for the UDP protocol. Each figure consists of two types of graphs. The $x$-axis in both graph types represents the sending speed and is measured as kilobytes per second. The $y$-axis of Graphs $1 a-4 a$ represents the receiving speed and is measured as kilobytes per second, as well. The $y$-axis of Graphs $1 b-4 b$ represents the percentage of received data. This type of graph is useful in finding the threshold at which data transmission speed starts to decrease significantly.

The graphs in Figures $1 a$ and $1 b$ show that the Celeron system is able to use the full capacity of $10 \mathrm{Mb} / \mathrm{s}$ Ethernet with data packets of 500 bytes. The Pentium 133 system uses approximately half of the bandwidth, while the 486 system shows poor results using about $10 \%$ of the bandwidth. The comparison indicates that the data transmission speed largely depends on the hardware configuration, especially on the processor speed.

The graphs in Figures $2 a$ and $2 b$ show significantly lower speed for the data load of two bytes. Low speed is caused by large message header overhead. The header for UDP packets used in tests consists of 8 bytes plus 20 bytes for an IP header. Total size of IP and UDP headers is 28 bytes, making the headers 14 times larger than the data load. Sending and receiving small packets also causes frequent operating system calls that additionally slow down the communication speed. The Celeron system running at $300 \mathrm{MHz}$ has the maximum data transmission speed of $6 \mathrm{kB} / \mathrm{s}$. It is about 2.1 times faster than the Pentium system at $133 \mathrm{MHz}$, and about 3.4 times faster than the 486 system at $66 \mathrm{MHz}$.

Figures $3 a$ and $3 b$ compare the three operating systems using 500 bytes of packet load. Linux is about two times faster than the Windows systems and is able to use the full capacity of $10 \mathrm{Mb} / \mathrm{s}$ Ethernet. The Windows 95 curve shows large deviation in receiving speed that occurs at $500 \mathrm{kB} / \mathrm{s}$. For a data transmission speed of 550 $\mathrm{kB} / \mathrm{s}$ and higher, Windows 95 shows better results than Windows NT.

The difference between Linux and Windows systems is larger for the data load of two bytes. This is presented 


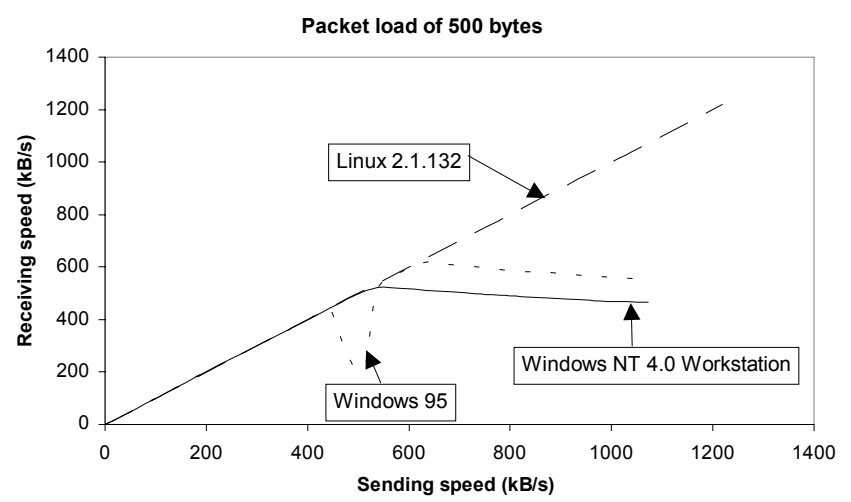

(a)

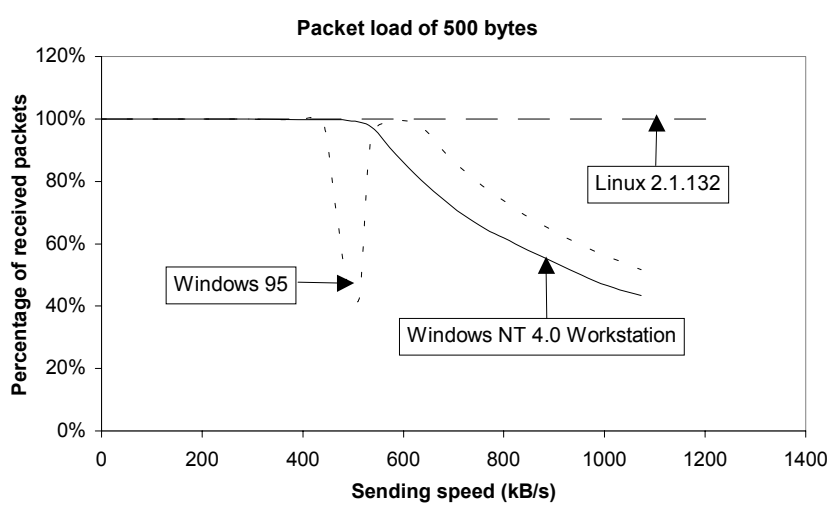

(b)

Figure 3: The comparison of UDP transmission speed for different operating systems and 500 bytes of packet load

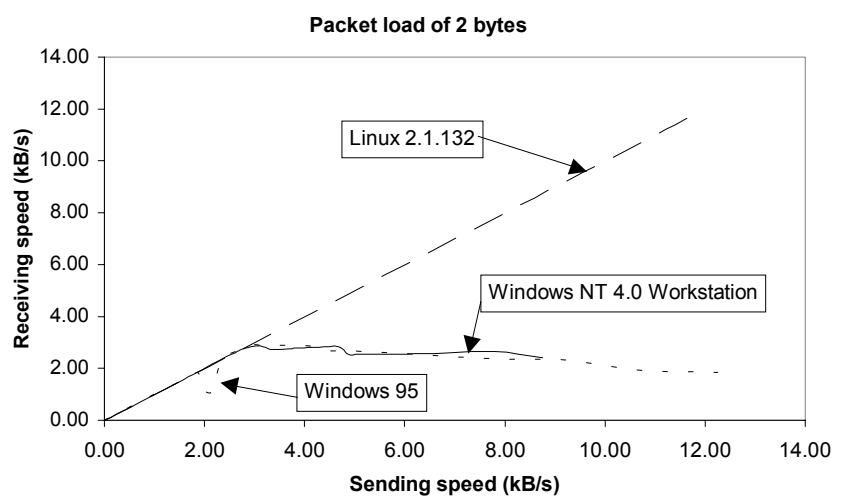

(a)

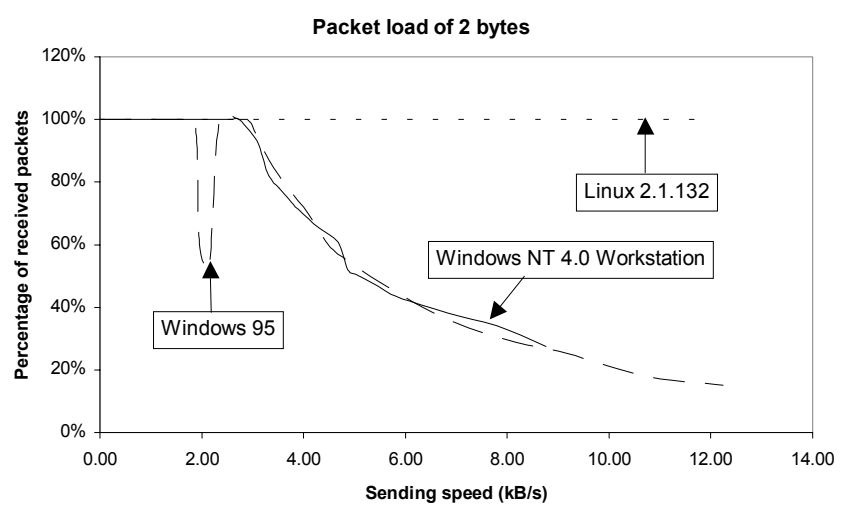

(b)

Figure 4: The comparison of UDP transmission speed for different operating systems and 2 bytes of packet load

in Figures $4 a$ and $4 b$. The Linux curve in Figures $4 a$ and $4 b$ has no threshold, because we could not reach the threshold with the sending machine we used. In our measurements Linux greatly outperformed the Windows systems with at least four times better results, but the actual maximum speed of Linux might be even higher. Except for a significant deviation of Windows 95 curve at $2 \mathrm{kB} / \mathrm{s}$, the characteristics for Windows NT 4.0 and Windows 95 are similar.

\section{TCP MEASUREMENT RESULTS}

One graph is sufficient to present the measurement results for TCP, as shown in Figures 5-8. Since TCP is a reliable protocol, the receiving speed is always equal to the sending speed. Therefore, graphs in Figures 5-8 consist of bars, where each bar represents the maximum receiving speed for the given configuration. Since TCP is a data stream oriented protocol, it packs the sequence of small data portions in one large TCP segment whose size depends on internal algorithms. Thus, the data transmission speed of small messages is higher for TCP than for UDP, which is evident if Figures 6 and 8 are compared with Figures 2 and 4.

In Figure 5, the results for reliable TCP communication with messages of 500 bytes are compared. The Celeron system is able to achieve the full speed of the TCP communication on $10 \mathrm{Mb} / \mathrm{s}$ Ethernet.
The Celeron machine also has $15 \%$ better results than the Pentium system, and twice the speed of the 486 system.

Figure 6 shows the maximum speed of the three systems with the message size of two bytes. The maximum speed obtained in this measurement is significantly lower than before. The Celeron system is almost twice as fast as the Pentium machine and about three times faster than the 486 machine. Since the Celeron machine in the previous test reaches the maximum capacity of the underlying network, the difference between the Celeron and the other systems in this test case is larger than before.

Figure 7 compares different operating systems for the message size of 500 bytes. Linux is able to use the whole bandwidth, while the Windows systems have 15\% lower data transmission speed. Comparing the two Windows systems, they show similar results, with Windows NT having 4\% lower speed than Windows 95.

The results of measurement with two-byte messages are presented in Figure 8. The Linux system has approximately $11 \%$ better results than both Windows systems. Windows 95 has higher data transmission speed than Windows NT 4.0, but the difference is less than $4 \%$. 


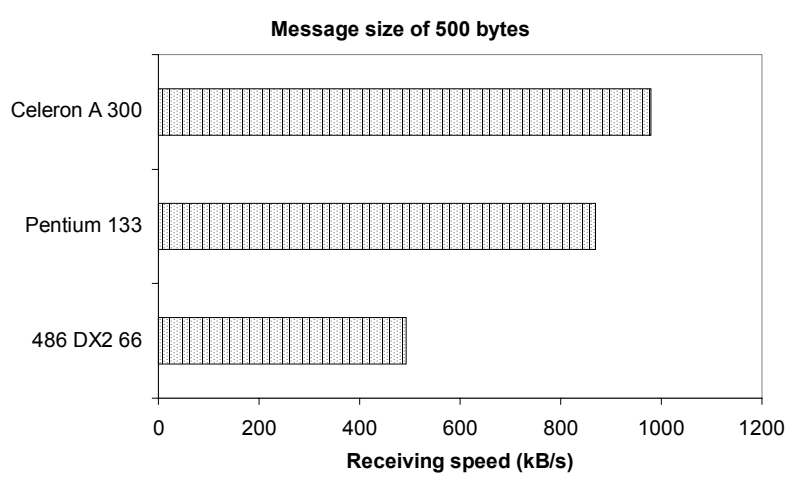

Figure 5: The comparison of TCP transmission speed for different hardware configurations of PCs and 500-byte messages

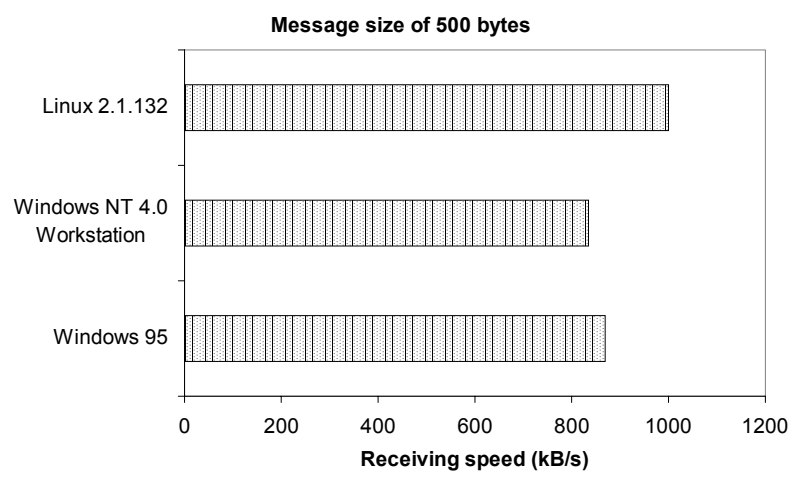

Figure 7: The comparison of TCP transmission speed for different operating systems and 500-byte messages

\section{CONCLUSION}

In this paper, we describe the set of measurements based on personal computers and $10 \mathrm{Mb} / \mathrm{s}$ Ethernet. We measured the data transmission speed between personal computers for two basic Internet data protocols, UDP and TCP. Different hardware configurations of PCs, different operating systems as well as two message sizes were used to find out how each of these parameters affects the communication speed.

Our results show that the hardware configuration significantly affects the communication speed of both UDP and TCP. Of the three tested hardware configurations, only the Celeron system is able to use the full capacity of $10 \mathrm{Mb} / \mathrm{s}$ Ethernet with 500-byte messages. The Pentium system and the 486 system have significantly lower speed. All tested systems achieved much higher data transmission speed with the larger messages. However, the difference between tested hardware configurations is larger for smaller data loads.

The comparison of operating systems for UDP and TCP communication shows that Linux outperforms both Windows systems. Windows 95 and Windows NT have similar results, with Windows 95 being slightly faster. In both UDP and TCP communication Linux uses the full capacity of $10 \mathrm{Mb} / \mathrm{s}$ Ethernet with larger messages. Though the Linux system is significantly faster than the Windows systems in UDP communication, the difference between these systems becomes smaller with TCP communication.

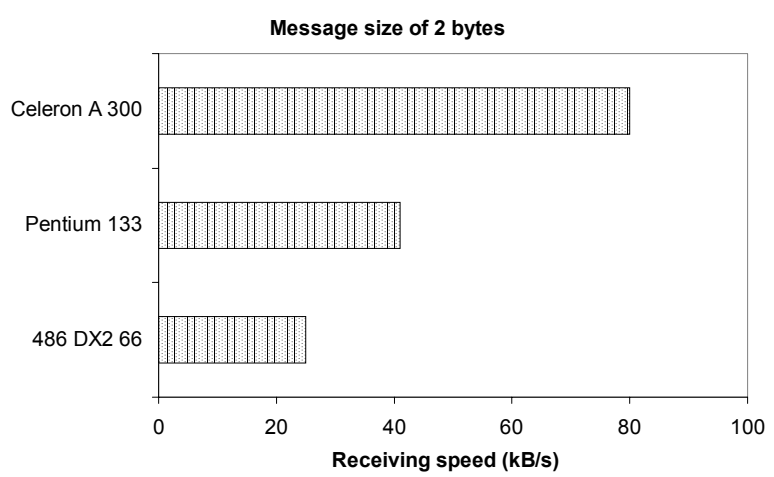

Figure 6: The comparison of TCP transmission speed for different hardware configurations of PCs and 2-byte messages

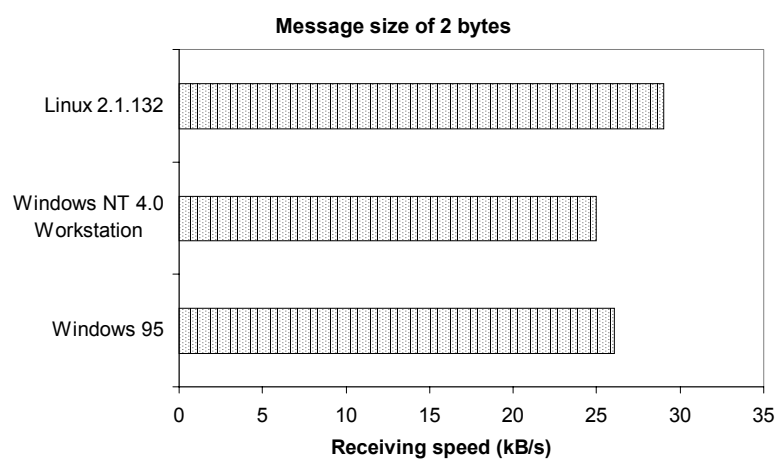

Figure 8: The comparison of TCP transmission speed for different operating systems and 2-byte messages

This paper shows that for small messages it is necessary to use very fast personal computer systems and real-time capable operating systems, in order to use the whole capacity of high-speed local networks. With larger data loads it becomes possible to use more of the network bandwidth. The paper also shows that the requirements for personal computers become less demanding if larger messages are used in communication between PCs.

\section{ACKNOWLEDGMENT}

The authors wish to thank A. Grbic, Željka Mihajlović, and Leonardo Jelenković for providing valuable comments on the research presented in this paper

\section{REFERENCES}

[1] A. Milanović, S. Srbljić and J. Radej: " Performance of Distributed Systems Based on Ethernet and Personal Computers ", Proceedings of ISIE '99, Bled, Slovenia, 1999.

[2] Chandramonah A. Thekkath and Henry M. Levy: "Limits to Low-Latency Communication on High-Speed Networks", $A C M$ Transactions on Computer Systems, Vol. 11, No. 2, May 1993, pp. 179-203.

[3] Douglas E. Comer: "Internetworking with TCP/IP", Vol. 1, Prentice Hall Inc., 1991

[4] T. Socolofsky, C. Kale: “A TCP/IP Tutorial”, RFC 1180 , Spider Systems Limited, January 1991

[5] R. Fielding, et. al: "Hypertext Transfer Protocol HTTP/1.1", RFC 2068, UC Irvine, January 1997 